\title{
Avaliação das indicações de ceratoplastia penetrante no interior paulista
}

\author{
Evaluation of penetrating keratoplasty indications \\ in inner part of the São Paulo state
}

Amanda Pires Barbosa ${ }^{1}$, Gildásio Castello de Almeida Júnior ${ }^{2}$, Marta Ferrari Teixeira ${ }^{3}$, José Carlos Barbosa ${ }^{4}$

\begin{abstract}
RESUMO
Objetivo: Determinar os diagnósticos clínicos corneanos na indicação da Ceratoplastia Penetrante (CP) de pacientes inscritos no Banco de Olhos do Hospital de Base de São José do Rio Preto, correlacionando-os com outras variáveis, como a faixa etária, sexo e situação sócioeconômica. Métodos:Realizou-se análise retrospectiva de 1085 fichas de inscrição de pacientes submetidos à Ceratoplastia Penetrante, no Hospital de Base de São José do Rio Preto, no período de 2000 a 2009. Os diagnósticos para indicação das cirurgias foram posteriormente correlacionados com a faixa etária, o sexo e o nível socioeconômico dos pacientes, por meio do teste do Quiquadrado. Resultados: Do total de Ceratoplastias Penetrantes, $57,4 \%$ foram realizadas em pacientes do sexo masculino, e a média de idade encontrada foi de 52,3 anos ( $\mathrm{DP}=21,42)$. Verificou-se que em $90,8 \%$ dos casos a cirurgia ocorreu por meio do Sistema Único de Saúde (SUS), enquanto nos demais $(9,2 \%)$ os pacientes possuíam convênio ou custearam todo o procedimento. A indicação mais frequentemente encontrada foi o leucoma (23\%), o qual ocorreu em 14\% das CPs realizadas em homens e em $9 \%$ das ocorridas em mulheres. Em relação às CPs com este diagnóstico, $40 \%$ ocorreram em pacientes com idade superior a 60 anos, com porcentagem decrescendo com as faixas etárias mais jovens, e 94,4\% efetuaram-se através do SUS, enquanto que apenas 5,6\% dos casos se encaixaram no quesito convênio/particular. Conclusão: A principal indicação à Ceratoplastia Penetrante encontrada foi o leucoma, o qual predominou em homens, nas idades acima de 60 anos, em pacientes do SUS.
\end{abstract}

Descritores: Transplante de córnea; Ceratoplastia penetrante; Doenças da córnea; Opacidade da córnea; Estudos retrospectivos

\begin{abstract}
Objective: To determinate the corneal diagnoses of patients underwent Penetrating Keratoplasty (PK) in the university hospital of São José do Rio Preto, São Paulo, Brazil, and their relation to age group, gender and socioeconomic factors. Methods: Retrospective analysis of 1085 registration forms of patients underwent Penetrating Keratoplasty in the university hospital of São José do Rio Preto, São Paulo, Brazil, from 2000 to 2009. The surgical indications were then correlated to age group, gender and socioeconomic factors, by chi-square test. Results: Fifty-seven percent of all Penetrating Keratoplasties were performed in male patients, and the mean age was 52.3 years. Most surgeries $(90.8 \%)$ occurred by means of public health care (SUS). The main diagnosis was leukoma $(23 \%)$, representing $14 \%$ of the indications for PK in males and 9\% in females. Leukoma was also the diagnosis in $40 \%$ of PK performed in patients older than 60 years and in $94.4 \%$ of these surgeries in public health care users. Conclusion: The main indication for Penetrating Keratoplasty was leukoma, predominating in males, ages above 60 years and in public health care users.
\end{abstract}

Keywords: Corneal transplantation; Keratoplasty, penetrating; Corneal diseases; Corneal opacity; Retrospective studies

\footnotetext{
${ }^{1}$ Curso Acadêmico da Faculdade de Medicina de São José do Rio Preto (FAMERP) - São José do Rio Preto (SP), Brasil;

${ }^{2}$ Fundação Faculdade Regional de Medicina de São José do Rio Preto - São José do Rio Preto (SP), Brasil;

${ }_{3}^{3}$ Departamento de Especialidades Cirúrgicas da Disciplina de Oftalmologia da Faculdade de Medicina de São José do Rio Preto (FAMERP) -

São José do Rio Preto (SP), Brasil;

${ }^{4}$ Departamento de Ciências Exatas, Universidade Estadual Paulista "Júlio de Mesquita Filho" (UNESP - Jaboticabal (SP), Brasil.

Departamento de Especialidades Cirúrgicas, Disciplina de Oftalmologia, da Faculdade de Medicina de São José do Rio Preto e Banco de Olhos do Hemocentro do Hospital de Base - São José do Rio Preto (SP) - Brasil.
}

Os autores declaram não haver conflitos de interesse

Recebido para publicação em: 10/8/2011 - Aceito para publicação em: 18/06/2012 


\section{INTRODUÇãO}

$\mathbf{O}$ transplante de córnea é uma das técnicas cirúrgicas mais antigas da oftalmologia ${ }^{(1)}$. Segundo dados do Ministério da Saúde, apenas no primeiro trimestre de 2009 foram realizados 6151 transplantes de córnea no Brasil, sendo que 2948 ocorreram no estado de São Paulo ${ }^{(2)}$. A melhor organização dos Bancos de Olhos, os quais proporcionam maior captação e preservação de córneas, a utilização de tecido doado meticulosamente selecionado graças ao desenvolvimento do microscópio especular e da contagem de células endoteliais, além do melhor conhecimento da fisiologia corneana e do uso de fios de sutura maleáveis, entre outros aperfeiçoamentos, possibilitaram a este procedimento sua realização rotineira e bem-sucedida ${ }^{(3-6)}$.

A ceratoplastia penetrante $(\mathrm{CP})$ geralmente é a cirurgia de escolha na reabilitação dos pacientes com cegueira provocada por doençascorneanas ${ }^{(7)}$, segunda causa de cegueira reversível no mundo, e que, por atingirem uma população jovem e ativa, causam importante perda econômica e social ${ }^{(8)}$. Entretanto, a prevalência das patologias da córnea difere entre os países e as populações, de acordo com as condições de saúde pública às quais a população está sujeita ${ }^{(8)}$.Nos EUA, a indicação mais frequente de CP é a ceratopatia bolhosa, enquanto na Europa e no Brasil os estudos apontam o ceratocone como a principal indicação, embora haja variações entre as diversas regiões desses países ${ }^{(9)}$.

Diante destes fatos, e considerando-se a importância representada pela ceratoplastia penetrante no que concerne à sua frequência ascendente, ao seu êxito e crescente aperfeiçoamento, e as triagens erroneamente feitas para doenças que às vezes não tem indicação para tal procedimento, torna-se relevante a análise de suas principais indicações pretendida por este estudo, assim como sua comparação com fatores como idade, sexo e nível socioeconômico.

\section{Métodos}

Efetuou-se estudo retrospectivo das fichas de indicação de 1116 Ceratoplastias Penetrantes realizadas em pacientes inscritos no Banco de Olhos do Hospital de Base (HB) de São José do Rio Preto, no período de 2000 a 2009, transplantados no referido hospital. Excluíram-se 31 fichas, cujo preenchimento incorreto gerou perda de informações necessárias à análise.

Obtiveram-se as indicações à $\mathrm{CP}$ descritas nas fichas, o sexo, a idade e o nível socioeconômico dos pacientes, sendo esta última variável analisada pelos seguintes critérios: paciente pertencente ao Sistema Único de Saúde (SUS) e aqueles vinculados a convênio ou a sistema particular. As idades foram classificadas em 4 faixas etárias, a saber: 0 a 19 anos, 20 a 39 anos, 40 a 59 anos, e 60 anos ou mais.

As indicações encontradas foram reunidas nos seguintes grupos: leucoma, ceratopatia bolhosa, ceratocone, rejeição, falência primária, ceratite bacteriana, ceratite viral, ceratite fúngica, distrofia de Fuchs, trauma ocular e "outras". Este termo inclui diagnósticos com paucidade de ocorrência.

Posteriormente, os diagnósticos corneanos foram relacionados com idade, sexo e nível socioeconômico dos pacientes em cada cirurgia realizada, por meio do teste Qui-quadrado, também utilizado para averiguar possíveis diferenças entre as frequências das indicações à $\mathrm{CP}$. Adotou-se $\mathrm{p}<0,05$ para rejeição da hipótese de nulidade. Os dados foram classificados em percentual de incidência e expostos em tabela e gráficos.

O estudo obteve a aprovação do Comitê de Ética em Pesquisa sob o protocolo de número 3378/2010.

\section{Resultados}

Das 1085 ceratoplastias penetrantes ocorridas no Hospital de Base de São José do Rio Preto, no período de 2000 a 2009, 623 $(57,4 \%)$ foram realizadas no sexo masculino e $462(42,6 \%)$ no sexo feminino. A média de idade encontrada foi de 52,3 anos $(\mathrm{DP}=21,42)$, com idades variando de 1 a 90 anos. Em 985 casos $(90,8 \%)$ a cirurgia ocorreu em pacientes do SUS, e apenas 100 $(9,2 \%)$ foram efetuadas por meio de convênio pago/particular.

A principal indicação encontrada (tabela 1) foi o leucoma, correspondendo a 250 casos $(23,04 \%)$, seguido por ceratopatiabolhosa (213 casos - 19,63\%), ceratocone (177 casos - 16,31\%), rejeição (144 casos - 13,27\%), falência primária e ceratite bacteriana (ambas com 69 casos - 6,36\%), ceratite viral (52 casos $-4,79 \%$ ), outras ( 38 casos $-3,50 \%$ ), distrofia de Fuchs ( 35 casos - 3,23\%), trauma ocular (23 casos - 2,12\%) e ceratitefúngica (15 casos - 1,38\%).

Os gráficos 1, 2 e 3 evidenciam a correlação das indicações de CP com o sexo, as faixas etárias e o nível socioeconômico dos pacientes, respectivamente. Em relação as $250 \mathrm{CPs}$ por leucoma, $152(60,8 \%)$ foram realizadas em pacientes do sexo masculino, 98 $(39,2 \%)$ no feminino, e $236(94,4 \%)$ ocorreram por meio do SUS, contra apenas 14 (5,6\%) do convênio/particular. Observaram-se 26 transplantes por leucoma na faixa etária de 0 a 19 anos (10,4\%), 52 dos 20 aos 39 anos (20,8\%), 72 dos 40 aos 59 anos (28,8\%) e 100 na faixa etária acima de 60 anos $(40 \%)$.

Tabela 1

Distribuição dos diagnósticos clínicos dos pacientes submetidos à ceratoplastia penetrante no período de 2000 a 2009 no Hospital de base de São José do Rio Preto

\begin{tabular}{lrr}
\hline Diagnóstico clínico & N & f (\%) \\
\hline Leucoma & 250 & 23,04 \\
Ceratopatia bolhosa & 213 & 19,63 \\
Ceratocone & 177 & 16,31 \\
Rejeição & 144 & 13,27 \\
Ceratite bacteriana & 69 & 6,36 \\
Falência primária & 69 & 6,36 \\
Ceratite viral & 52 & 4,79 \\
Outras & 38 & 3,50 \\
Distrofia de Fuchs & 35 & 3,23 \\
Trauma ocular & 23 & 2,12 \\
Ceratitefúngica & 15 & 1,38 \\
\hline
\end{tabular}

\section{Discussão}

Há numerosos relatos referentes às indicações de $\mathrm{CP}$, os quais mostram sua variedade nas diversas partes do mundo. Estudos desenvolvidos nos EUA, Canadá, Singapura e Japão têm documentado ser a ceratopatia bolhosa do pseudofácico a principal indicação à CP. Avaliações realizadas na Austrália, Nova Zelândia, Kênia, e Europa, na mesma época, apontam o ceratocone como a indicação mais frequente em suas amostras observadas. Em recente pesquisa realizada em hospital terciário na China, demonstrou-se que a ceratite herpética $(24,1 \%)$ e o trauma ocular $(21,2 \%)$ são os diagnósticos mais recorrentes indicados a este tipo de transplante ${ }^{(7)}$.

No Brasil, a principal indicação de CP é o ceratocone, explicada por alguns autores pelos ótimos resultados que se obtém com esse tratamento, seguido da ceratopatia bolhosa ${ }^{(9-12)}$. Entretanto, diferentes regiões apresentam espectros de doen- 


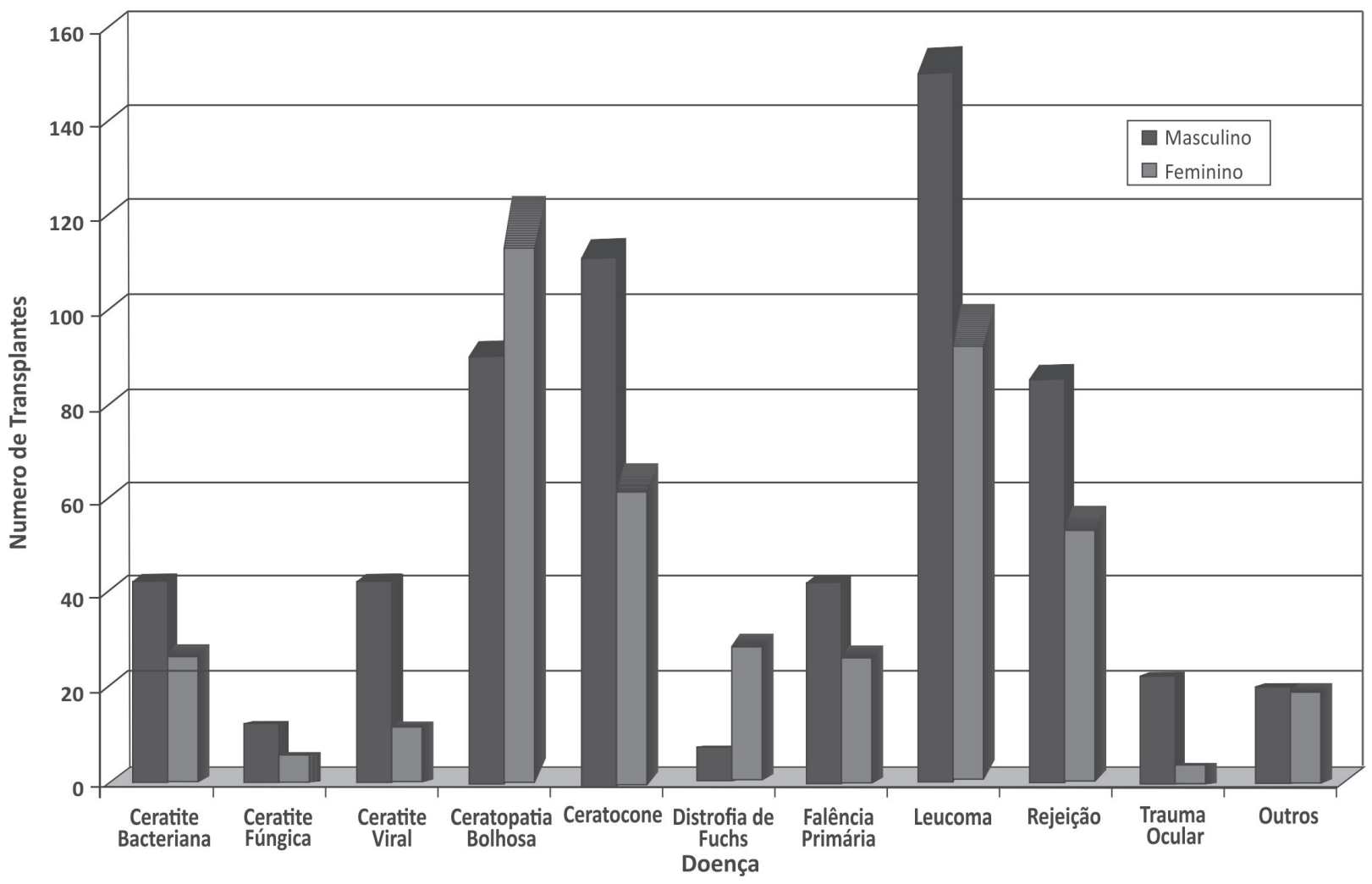

Gráfico 1: Correlação entre as indicações de ceratoplastia penetrante e o sexo dos pacientes

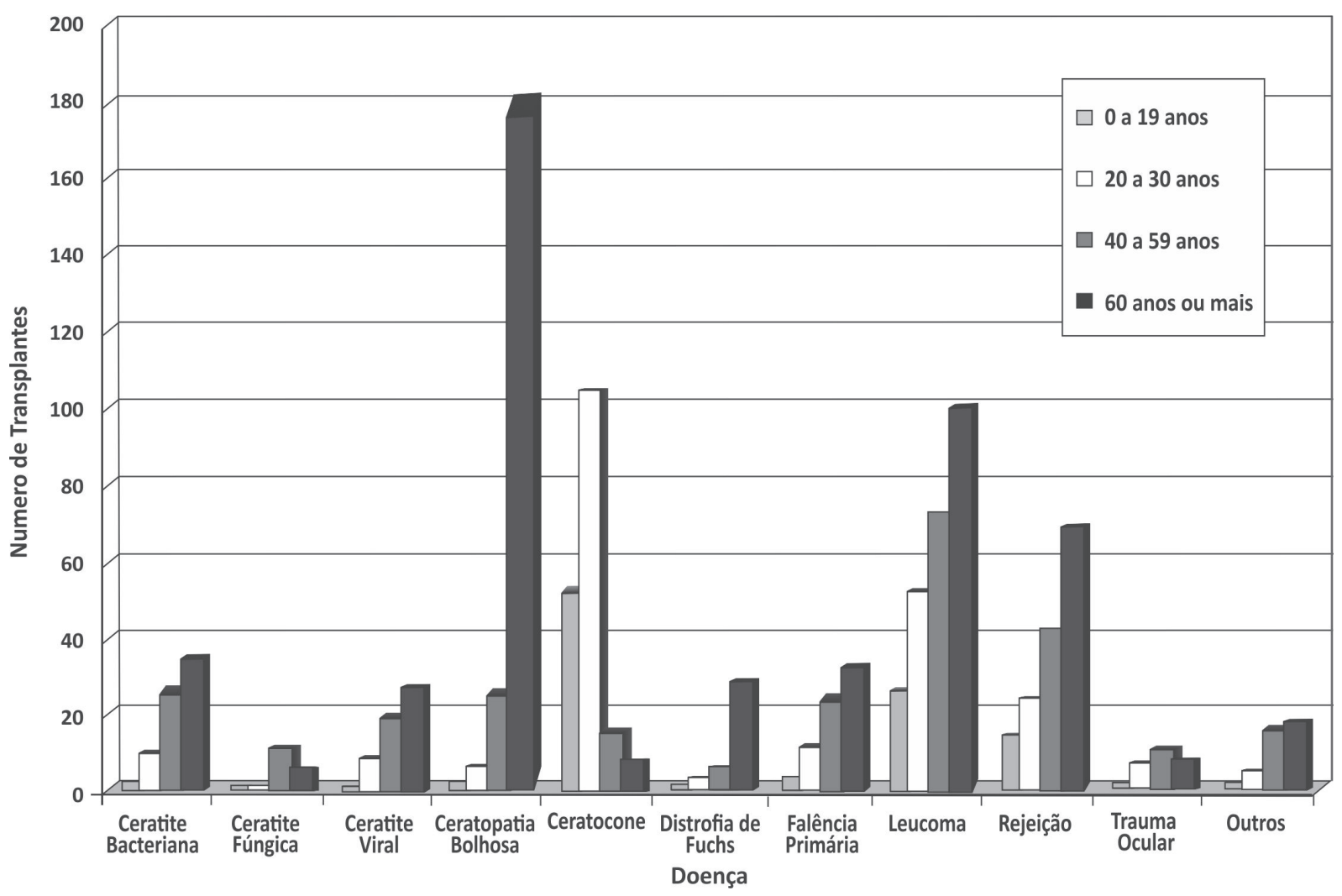

Gráfico 2: Correlação entre as indicações de ceratoplastia penetrante e a faixa etária dos pacientes 


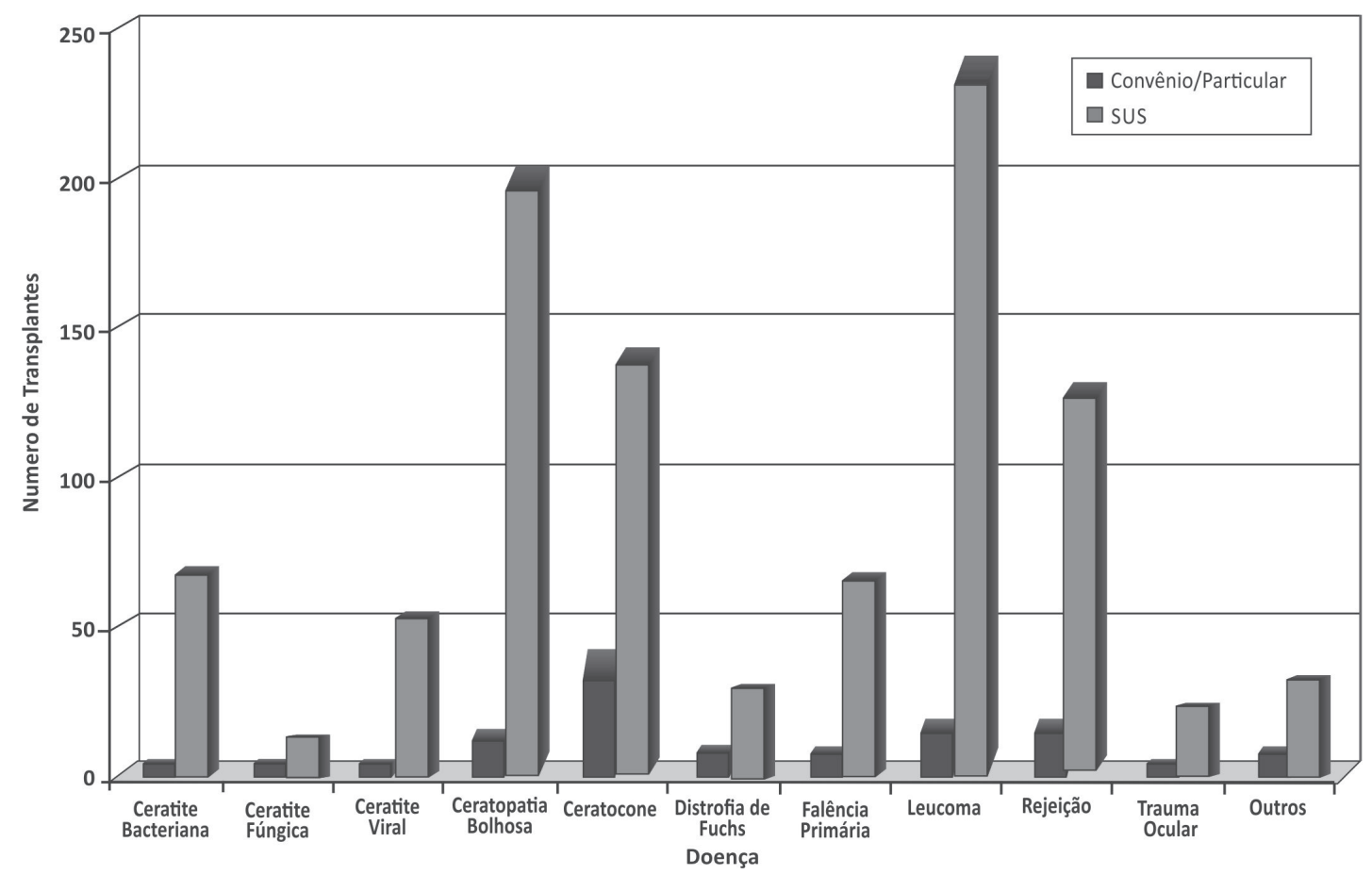

Gráfico 3: Correlação entre as indicações de ceratoplastia penetrante e o nível socioeconômico dos pacientes

ças corneanas distintos, o que contribui para variações nas indicações de CP. Em nosso estudo, as patologias mais indicadas ao transplante penetrante foram leucoma $(23,04 \%)$, ceratopatiabolhosa $(19,63 \%)$ e ceratocone $(16,31 \%)$, mantendo a tendência já apresentada em outro trabalho realizado no Hospital de base de São José do Rio Preto, nos anos de 1991 a 1998, cujo resultado foi $53,4 \%$ de CPs por leucoma, seguido por ceratopatia bolhosa do pseudofácico $(13,8 \%)$, e ceratocone $(11,3 \%)^{(13)}$. Em estudo estabelecido em hospital deMinas Gerais, a indicação mais comum de CP foi a lesão ulcerativa ${ }^{(14)}$, em concordância com a Santa Casa de São Paulo (84,7\% de ceratites infecciosas) $)^{(15)}$, no mesmo período, e com hospitais de Pernambuco e do Amazonas $^{(16)}$.Por outro lado, em Sergipe notou-se predomínio da ceratopatia bolhosa ${ }^{(17)}$, enquanto Porto Alegre corroborou a indicação dominante no Brasil, o ceratocone ${ }^{(10)}$.

Em estudos prévios encontrou-se predomínio de CPs no sexo masculino ${ }^{(9,11-17)}$, em concordância com o observado por nós (57,4\%), embora outras instituições apresentem resultados diferentes ${ }^{(3)}$. A média de idade em nosso estudo foi de 52,3 anos, semelhante à ocorrida nos demais trabalhos ${ }^{(11,13-17)}$, à exceção daqueles cuja principal indicação foi o ceratocone ${ }^{(9,12)}$, em que a média obtida foi menor.

Em concordância com estudo realizado em Taiwan ${ }^{(18)}$ e no norte da Índia ${ }^{(19)}$, o leucoma foi diagnóstico mais recorrente, possivelmente pelo fato de a maioria dos pacientes serem do SUS. Existem trabalhos na Índia, nos quais o leucoma também foi a indicação mais frequente de CPs, que correlacionam esta causa a problemas decorrentes de acessibilidade e disponibilidade dos cuidados oftalmológicos. Tais condições são prevalentes nos países subdesenvolvidos, devido ao diagnóstico tardio de infecções corneanas, comconsequente evolução para a opacidade desse tecido.Os pacientes com status socioeconômico baixo estão mais suscetíveis às infecções corneanas e ao desenvolvimento do leucoma, e tem menor acesso a tratamentos que previnam a necessidade de transplante ${ }^{(19,20)}$.

Observou-se predomínio do leucoma nas CPs realizadas em homens, os quais são mais expostos às lesões que originam o leucoma, como traumas e ceratites. Houve aumento de sua frequência progressivamente com o aumento da idade, sendo a maior causa de CPs na faixas etárias de 40 a 59 anos e de 60 anos ou mais, o que pode se justificar pela existência de condições que aumentam a chance de leucoma em nosso meio nessa faixa etária, como a opacidade corneana tracomatosa ${ }^{(21)}$.

A ceratopatia bolhosa surgiu como a segunda maior causa de CPs realizadas em nosso serviço, reflexo da popularização da cirurgia para correção de catarata, em especial a facoemulsificação, e do aumento dos implantes intraoculares ${ }^{(9)}$, que tem como principal complicação a ceratopatia bolhosa do pseudofácico. As CPs decorrentes de ceratopatia bolhosa ocorreram principalmente em indivíduos com 60 anos ou mais, idade em que a população é mais acometida pela catarata e, portanto, a incidência de cirurgia para corrigi-la e de suas complicações é também maior.

A terceira posição ocupada pelo ceratocone em nossa amostra pode ser explicada pela melhora nas técnicas de adaptação e fabricação de lentes de contato ${ }^{(3,14)}$, bem como a maior incidência, neste estudo, de CP em idades acima de 60 anos (504 casos - $46,45 \%$ ), uma vez que o ceratocone predomina em faixas etárias mais jovens. Pesquisas ocorridas em Israel e na Alemanha, cujos resultados apontam o ceratocone como a principal indicação à $\mathrm{CP}$, atribuem as características demográficas e genéticas às diferenças entre os países ${ }^{(22,23)}$.O número de CPs por ceratocone em nossa análise foi acentuadamente superior nas idades de 0 a 19 anos ( 52 casos em $177-29,37 \%$ ), e de 20 a 39 anos (103 casos em $177-58,19 \%)$, as quais representaram apenas $9,22 \%$ e $20,55 \%$, respectivamente, do total de CPs.

Os retransplantes estão entre causas importantes de CPs, o que corrobora outros estudos ${ }^{(3,10,12,18,23,24)}$, sendo um reflexo do número crescente de receptores de transplante penetrante de córnea, pelo maior acesso a córneas doadoras possibilitado pela atividade dos Bancos de Olhos.

As ceratites infecciosas de nossa amostra totalizam uma porcentagem inferior às apresentadas em Minas Gerais ${ }^{(14)}$, Pernambuco $^{(16)}$, Israel(23), Índia ${ }^{(20)}$, cujos autores relacionam o elevado contingente representado por este diagnóstico às más condições socioeconômicas da maior parte dos pacientes incluídos nestes estudos, bem como à dificuldade no diagnóstico e tratamento precoce dos agentes infecciosos. 
A Distrofia de Fuchs apresenta-se como uma patologia rara, tanto em nosso grupo estudado $(3,23 \%)$, quanto nos diversos trabalhos realizados ${ }^{(7,18,20,22)}$, sendo explicada por causas genéticas. Trauma também mostrou-se uma indicação infrequente nesta pesquisa $(2,12 \%)$, assim como em diversos estudos analisados ${ }^{(9,14,23,24)}$, ao contrário de outros trabalhos, em que esta foi a segunda maior causa de $\mathrm{CP}^{(3,7)}$.

A faixa etária prevalente observada, de 60 anos ou mais, é consequência do aumento da expectativa de vida brasileira, da maior conscientização dos pacientes quanto ao tratamento e à prevenção de doenças oculares, entre outras causas. Em relação ao nível socioeconômico dos pacientes, pode-se concluir que, por se tratar de um hospital-escola, é esperado encontrar-se uma frequência aumentada de CPs em pacientes do SUS (985 casos - 90,78\%), o que limita as correlações entre esta variável e as demais feitas pelo presente estudo.

Considerando-se que o leucoma é, na realidade, a consequência final de diversas desordens, como tracoma, trauma e ceratite infecciosa, conclui-se que os resultados deste estudo apresentam limitações, decorrentes da dificuldade em se definir a etiologia do leucoma.Além disso, é previsível que as indicações de transplante de córnea sejam alteradas continuamente, por influência de inúmeros fatores, como a tendência de aumento de doadores devido a maior divulgação e conscientização da população, o aumento do número de banco de olhos no Brasil, o aperfeiçoamento da técnica cirúrgica e dos meios de preservação das córneas ${ }^{(12)}$.

\section{CONCLUSÃO}

As principais indicações de ceratoplastia penetrante observadas em nosso estudo são o leucoma (23,04\%), a ceratopatia bolhosa $(19,63 \%)$ e o ceratocone $(16,31 \%)$. Houve predomínio das CPs por leucoma em homens $(60,8 \%)$, na faixa etária acima de 60 anos (40\%), em pacientes do SUS (94,4\%).

Para melhor avaliar estas relações, futuros estudos comparando as causas de CPs com a o sexo, a faixa etária e o nível socioeconômico são necessários, em especial no que concerne a esta última variável, uma vez que o presente estudo foi realizado em um hospital-escola, em que o número de cirurgias realizadas pelo SUS supera em muito o do sistema particular/convênio.

\section{Agradecimentos}

Agradecemos à fonte financiadora deste estudo, Bolsa de Iniciação Científica(BIC) da Faculdade de Medicina de Rio Preto (FAMERP), durante o período de 2010 a 2011.

\section{REFERÊNCIAS}

1. Garralda A, Epelde A, Iturralde O, Compains E, Maison C, Altarriba M, et al. Transplante de córnea. AnSistSanitNavar. 2006;29(Supl2):163-74.

2. Ministério da Saúde. Portal da Saúde. Transplantes de órgãos crescem 24,3\%(Internet)(citado em 2009 Setembro 25). Disponível em: http://portal.saude.gov.br/portal/aplicacoes/ noticias/default.cfm?pg= dspDetalhe Noticia\& id_area $=124$ $\& \mathrm{CO}_{-}$NOTICIA $=10592$.

3. Sano FT, Dantas PEC, Silvino WR, Sanchez JZ, Sano RY, Adams F, Nishiwaki-Dantas MC. Tendência de mudança nas indicações de transplante penetrante de córnea. ArqBras Oftalmol. 2008;71(3):400-4

4. Almeida Junior GC, Watanabe KP, Teixeira MF, Cordeiro JA Influência da causa do óbito, idade do doador e tempo depreservação da córnea na contagem de células endoteliais num Banco de Olhos vinculado a um hospital escola. ArqCiênc Saúde. 2007;14(3):140-4.
5. Moffatt SL, Cartwright VA, Stumpf TH. Centennial review of corneal transplantation.Clin Experiment Ophthalmol. 2005;33(6): 642-57.Comment inClin Experiment Ophthalmol. 2006;34(4):3878.

6. RahmanI, Carley F, Hillarby C, Brahma A, Tullo AB. Penetrating keratoplasty: indications, outcomes, and complications. Eye(Lond). 2009;23(6):1288-94.

7. Pan $\mathrm{Q}, \mathrm{Li} \mathrm{X}, \mathrm{Gu} \mathrm{Y}$. Indications and outcomes of penetrating keratoplasty in a tertiary hospital in the developing world.Clin Exp Ophthalmol.2011 May 17. [Epub ahead of print].

8. Adán CBD, Diniz AR, Sato EH. Dez anos de doação de córneas no Banco de Olhos do Hospital São Paulo: perfil dos doadores de 1996 a 2005. ArqBras Oftalmol. 2008;71(2):176-81.

9. Flores VGC, Dias HLR, Castro RS. Indicações para ceratoplastia penetrante no Hospital das Clínicas-UNICAMP. ArqBras Oftalmol. 2007;70(3):505-8.

10. Cattani S, Kwitko S, Kroeff MAH, Marinho D, Rymer S, Bocaccio FL.Indicações de transplante de córnea no Hospital de Clínicas de Porto Alegre.ArqBras Oftalmol. 2002;65(1):95-8.

11. Cavalcanti MTD, Mahon M, Nóbrega DAT, Remígio MCA, Pires CS. Ceratocone: resultados visuais, complicações e qualidade de vida após ceratoplastia penetrante realizada por médico residente.ArqBras Oftalmol. 2004:67(3):415-8

12. Calix Netto MJ, Giustina ED, Ramos GZ, Peccini RFC, Sobrinho M, Souza LB. Principais indicações de transplante penetrante de córnea em um serviço de referência no interior de São Paulo (Sorocaba - SP, Brasil). ArqBras Oftalmol. 2006; 69(5):661-4.

13. Teixeira MF, Almeida Júnior GC, Rodrigues ML, Kamimoto PS, Kashiwabuchi LK. Resultados e indicações de ceratoplastias penetrantes realizadas por médicos em treinamento, num país em desenvolvimento. ArqBras Oftalmol. 2001;64(6):557-61.

14. Neves RC, Boteon JE, Santiago APMS. Indicações de transplantede córnea no Hospital São Geraldo da Universidade Federal de Minas Gerais. RevBras Oftalmol. 2010;69(2):84-8.

15. Oliveira FC, Dantas PEC, Marco ES, Oliveira AC, Nishiwaki-Dantas MC. Transplante terapêutico de córnea: resultados prolongados de série de casos. ArqBras Oftalmol. 2007;70(4):625-31.

16. Amaral CSR, Duarte JY, Silva PLS, Valbuena R, CunhaF. Indicações de ceratoplastia penetrante em Pernambuco. ArqBras Oftalmol. 2005;68(5):635-7.

17. Araújo AA, Melo GB, Silva RL, Araújo Neta VM. Perfil epidemiológico dos pacientes na lista de espera para transplante de córnea no Estado de Sergipe. Arq Bras Oftalmol. 2004;67(4):613-6.

18. Chen WL, Hu FR, Wang IJ. Changing indications for penetrating keratoplasty inTaiwan from 1987 to 1999 . Cornea. 2001;20 (2):141-4.

19. Sony P, Sharma N, Sen S, Vajpayee RB. Indications of penetrating keratoplasty in northern India.Cornea. 2005;24(8):989-91.

20. Dandona L, Ragu K, Janarthanan M, Naduvilath TJ, Shenoy R, Rao GN. Indications for penetrating keratoplasty in India.Indian J Ophthalmol.1997;45(3):163-8.

21. Schellini SA, Kitakawa D, Onishi E, Hoyama E, Padovani CR. Prevalência de tracoma cicatricial em Botucatu - estado de São Paulo. RevBras Oftalmol. 2006;65(4):220-3.

22. Cursiefen C, Küchle M, NaumannGO. Changing indications for penetrating keratoplasty: histopathology of 1,250 corneal buttons. Cornea. 1998;17(5):468-70.

23. Yahalom C, Mechoulam H, Solomon A, Raiskup FD, Peer J, FruchtPery J. Forty years of changing indications in penetrating keratoplasty in Israel. Cornea. 2005;24(3):256-8.

24. Siganos CS, Tsiklis NS, Miltsakakis DG, Georgiadis NS, Georgiadou IN, Kymionis GD, Pallikaris IG. Changing indications for penetrating keratoplasty in Greece, 1982-2006: a multicenter study.Cornea. 2010;29(4):372-4.

\section{Autor Correspondente:}

Gildásio C. Almeida Júnior

Faculda de de Medicina de São José do Rio Preto, Departamento de Especialidades Cirúrgicas, Oftalmologia

Av. Brigadeiro Faria Lima, 5416 Vila São Pedro

CEP 15090-000 - São José do Rio Preto, SP, Brasil

Telefone: (17)3201-5700, Ramal 5725

E-mail: gcaj@uol.com.br 